\title{
Design of Pattern Classifiers Using Optimum-Path Forest with Applications in Image Analysis
}

\author{
Alexandre X. Falcão \\ Institute of Computing (IC) - University of Campinas (UNICAMP), Brazil \\ afalcao@ic.unicamp.br \\ http://www.ic.unicamp.br/ afalcao/
}

\begin{abstract}
Current image acquisition and storage technologies have provided large data sets (with millions of samples) for analysis. Samples may be images from an image database, objects extracted from several images, or image pixels. This scenario is very challenging for traditional machine learning and pattern recognition techniques, which need to be more efficient and effective in large data sets. This lecture presents a recent and successful methodology, which links training samples in a given feature space and exploits optimum connectivity between them to the design of pattern classifiers. The methodology essentially extends the Image Foresting Transform, successfully used for filtering, segmentation and shape description, from the image domain to the feature space. Several supervised and unsupervised learning techniques may be created from the specification of two parameters: an adjacency relation and a connectivity function. The adjacency relation defines which samples form arcs of a graph in the feature space.

The connectivity function assigns a value to any path in the graph. The path value indicates the strength of connectedness of its terminal node with respect to its source node. A connectivity map is maximized by partitioning the graph into an optimum-path forest rooted at its maxima (i.e., representative samples of each class/group, called prototypes). The optimum-path forest is then a pattern classifier, which assigns to any new sample the class (or group label) of its most strongly connected root. The methods have been successfully applied to several applications and this lecture demonstrates two recent ones: content-based image retrieval (CBIR) and 3D segmentation of brain tissues in MR images. In CBIR, user interaction is considerably reduced to a few clicks on relevant/irrelevant images along 3 iterations of relevance feedback followed by supervised learning in order to achieve satisfactory query results. The $3 \mathrm{D}$ segmentation of brain tissues is automatically obtained in less than 2 minutes. It exploits voxel clustering, some prior knowledge and does not require a brain atlas for that purpose, while many other brain tissue segmentation methods do. The lecture concludes by discussing some open problems and perspectives for the optimum-path forest classifiers.
\end{abstract}

